Leticia Suárez'

Catherine Menkes"

\section{Violencia familiar ejercida en contra de los adolescentes mexicanos}

\section{Domestic violence against Mexican teenagers}

\section{RESU MEN}

OBJETIVO: Analizar la percepción de los estudiantes en torno a la violencia intrafamiliar.

MÉTODOS: El estudio se realizó mediante una muestra aleatoria representativa $(n=5,982)$ de estudiantes de secundaria y preparatoria de 13 a 19 años de edad en Chiapas y en San Luis Potosí, México, en el año 2003. Los estudiantes respondieron a un cuestionario de autollenado acerca de temas de educación sexual, sexualidad, embarazo y comportamientos de riesgo de los adolescentes y violencia intrafamiliar. Considerando el efecto del diseño muestral, se efectuó un análisis de regresión logística para ver asociación entre características sociodemoeconómicas y el riesgo de sufrir una agresión en el hogar.

RESULTADOS: El análisis estadístico multivariado confirma la mayor vulnerabilidad femenina frente a la violencia familiar en ambas entidades federativas (Chiapas: $\mathrm{RM}=1.87, \mathrm{p}=0.01$; San Luis: $\mathrm{RM}=1.53, \mathrm{p}=0.00$ ). Asimismo, el que los estudiantes bebieran alcohol (Chiapas: $R M=2.52, p=0.00$; San Luis: $R M=1.93, p=0.00$ ) o consumieran drogas (Chiapas: $\mathrm{RM}=2.27, \mathrm{p}=0.02$; San Luis: $\mathrm{RM}=1.82, \mathrm{p}=0.01$ ) resultaron ser las dos variables más importantes que expusieron a los estudiantes a la violencia intrafamiliar. Se mostró que la interacción del consumo de alcohol con la edad de los estudiantes aumentó el riesgo de violencia. Así, los más jóvenes tuvieron un riesgo del $51 \%$ en Chiapas y del $73 \%$ en San Luis de sufrir una agresión de un familiar que los de mayor edad.

CONCLUSIONES: Los adolescentes continúan siendo una de las poblaciones más vulnerables debido a su dependencia física, afectiva, económica y social hacia los adultos a su cargo. Esta encuesta es una de las primeras aproximaciones a la medición de violencia hacia los estudiantes.

Dirección de Salud Reproductiva. Centro de Investigación en Salud Poblacional. Instituto Nacional de Salud Pública. Cuernavaca, Morelos, México

" Centro Regional de Investigaciones Multidisciplinarias. Universidad Nacional Autónoma de México. Cuernavaca, Morelos, México

Correspondencia | Correspondence: Catherine Menkes

Centro Regional de Investigaciones Multidisciplinarias

Universidad Nacional Autónoma de México (CRIM/UNAM)

Priv. Chimalacatlán, 200 Col. Reforma C.P. 62220 , Cuernavaca, M orelos, México E-mail: menkes@servidor.unam.mx

Recibido: 30/3/2005 Revisado: 3/1/2006 Aprobado: 10/2/2006
DESCRIPTO RES: Violencia familiar, psicología. Comportamiento del adolescente. Factores de riesgo. Factores socioeconómicos. Vulnerabilidad en salud. México.

\section{ABSTRACT}

OBJECTIVE: To assess students' perception of domestic violence.

METHODS: The study was carried out in a random sample representative $(n=5,982)$ of high-school students aged between 13 and 19 years in Chiapas and San Luis Potosi, Mexico, in 2003. The students answered a self-administered questionnaire on sexual education, sexuality, pregnancy and risk behavior, and domestic violence. Considering the effects of the sample design, a logistic regression analysis was 
carried out in order to verify the association between socioeconomic and demographic characteristics and risk of domestic violence.

RESULTS: The multivariate statistical analysis confirmed that female students are more vulnerable to domestic violence in both states studied (Chiapas: $\mathrm{OR}=1.87, \mathrm{p}=0.01$; San Luis: $\mathrm{OR}=1.53, \mathrm{p}=0.00$ ). Moreover, alcohol (Chiapas: $\mathrm{OR}=2.52, \mathrm{p}=0.00$; San Luis: $\mathrm{OR}=1.93, \mathrm{p}=0.00$ ) or drug use (Chiapas: $\mathrm{OR}=2.27, \mathrm{p}=0.02$; $\mathrm{San}$ Luis: $\mathrm{OR}=1.82$, $\mathrm{p}=0.01$ ) were the two most significant variables for students exposure to domestic violence. The association between alcohol consumption and student's age increases the risk of violence. The youngest students had 51\% (Chiapas) and 73\% (San Luis) more risk of experience violence by a relative than older students.

CONCLUSIONS: Teenagers remain one of the most vulnerable population groups to domestic violence due to their physical, affective, economic and social dependence on other adults. The study was one of the first attempts to measure domestic violence against students.

\section{KEYW O RDS: Domestic violence, psychology. Adolescent behavior. Risk} factors. Socioeconomic factors. Health vulnerability. Mexico.

\section{INTRODUCCIÓN}

En fechas recientes la violencia intrafamiliar, definida como los actos violentos cometidos por un individuo o un pequeño grupo de individuos, ${ }^{15}$ se ha convertido en uno de los problemas fundamentales de la salud pública. Se trata de un fenómeno poco visible debido a la difícil denuncia de los hechos. Desde hace dos décadas este problema social empezó a cobrar visibilidad gracias a los movimientos sociales a favor de los derechos de las víctimas. Fue a partir de este momento que el tema de la violencia se ha incluido en las agendas de discusión internacional.* Desafortunadamente, el enfoque predominante se ha dirigido a la violencia entre cónyuges, cuando es igual de urgente centrarse también en la violencia que la familia ejerce contra los hijos.

Varias investigaciones ${ }^{3,11,23}$ señalan que la exposición constante de los jóvenes a la violencia familiar desde temprana edad tiene alcances devastadores en el desarrollo - cognitivo, afectivo y de relación - haciéndolos susceptibles a presentar síntomas que van desde dolores de cabeza o estómago, problemas de sueño, estrés y angustia hasta estados depresivos, psicóticos, presentando bajo rendimiento escolar, teniendo problemas de conducta y adicciones. Además de las consecuencias directas, varias estudios encuentran que los hijos repiten los patrones de violencia vividos en sus hogares. ${ }^{21}$ Los padres actúan como modelos de comportamientos violentos que los hijos observan y luego imitan. ${ }^{7,15}$ La literatura en general, confirma el hecho de que existe la transmisión intergeneracional de la violencia vivida en los hogares. ${ }^{5}$

También se han encontrado diferencias en la violencia intrafamiliar ejercida entre géneros. Aunque no hay un patrón uniforme, algunos estudios ${ }^{8}$ reportan una mayor agresión hacía mujeres adolescentes en comparación con los hombres. Una de las posibles explicaciones de este comportamiento se basa en una mayor preocupación de los padres sobre la autonomía de las hijas, especialmente en el aspecto de su sexualidad. Además, de que es más difícil maltratar a los varones por su mayor tamaño y fuerza. Una explicación alternativa es que los adolescentes del sexo masculino reportan menores agresiones debido a la gran aceptación social de los hombres envueltos en violencia. ${ }^{2}$ En sociedades tradicionales, los estereotipos de género, asocian a la mujer como un ser sometido y victimado, y al hombre como naturalmente violento y con el privilegio de poder ejercer violencia. ${ }^{25}$ Los roles asociados a cada género no son cuestionados por gran parte de la población.******

Además de que las mujeres son las que sufren mayor violencia, tienden a repetir el mismo patrón hacia sus hijas. La literatura ha mostrado que la violencia de las madres hacia los hijos puede estar fuertemente permeada por la pobreza. El estrés y otros efectos que produce la penuria llevan, en especial, al uso de violencia de las madres hacia sus hijas. ${ }^{8}$

*O rganización de los Estados Americanos. Convención Interamericana para Prevenir, Sancionar y Erradicar la Violencia contra la Mujer, Belém do Pará. Brasil, 1994. Disponible en URL: http://www.oas.org/CIM/Spanish/ConvencionViolencia.htm [acceso en 5 ago 2006] **Barbieri T. La subordinación de las mujeres en una sociedad desigual. N otas para un diagnóstico de la condición femenina en México. Instituto de Investigaciones Sociales, UNAM; 1988.

***García B, De Oliveira O. Trabajo femenino y vida familiar en México. México: El Colegio de México; 1994. 
Aparte de que existe un consenso de que la violencia en general genera violencia, también se ha encontrado que los jóvenes con una historia de victimización son más propensos a comprometerse en una variedad de comportamientos riesgosos, ${ }^{9}$ tales como el abuso de alcohol y drogas cuando estos han sido maltratados por sus progenitores. ${ }^{4}$ El rechazo de los padres hacia los hijos y la violencia contra ellos se asocia con un mayor uso de alcohol. ${ }^{24}$

En lo que respecta a México, no existe en la actualidad una encuesta nacional dirigida a estudiar la violencia ejercida de padres a hijos, pese a que se ha reportado una alta incidencia de jóvenes que acuden a centros de salud por haber sido violentados al interior de la familia.* Por tanto, se vuelve evidente la necesidad primordial de realizar estudios que contemplen la violencia intrafamiliar y los factores que la determinan.

Así, el principal objetivo de este trabajo fue analizar la percepción de los estudiantes masculinos y femeninos, en torno a la violencia intrafamiliar.

\section{MÉTO DOS}

La investigación buscó estudiar la salud reproductiva de los adolescentes en México en el año 2003. Se efectuó un estudio descriptivo exploratorio de corte cuantitativo y se diseñó una encuesta para obtener un diagnóstico de la problemática.

La investigación se efectuó en dos entidades federativas de alto nivel de marginación económica y social: Chiapas y San Luis Potosí (SLP). Se escogieron estos dos lugares ya que, desde el punto de vista de salud reproductiva, presentan altas tasas de natalidad y mortalidad materno-infantil, entre otras. Además, de ser poblaciones eminentemente rurales; así, en Chiapas cerca del $70 \%$ de la población vivía, en 2003, en localidades menores a 15,000 habitantes. SLP también presentaba un elevado porcentaje de población rural (cerca del 50\%), porcentaje menor al de Chiapas, pero claramente mayor al que se observa en el ámbito nacional (33\%).**

La población de estudio fue constituida por los alumnos y alumnas de escuelas públicas que se encontraban cursando de segundo de secundaria al último año de preparatoria. Mediante una muestra aleatoria de las escuelas públicas en el ámbito estatal, se seleccionó aleatoriamente el grado escolar y el grupo a ser entrevistado en cada escuela. Para lograr la representatividad estatal se seleccionaron 80 escuelas en cada estado.

El cuestionario fue aprobado por cada uno de los directores de las escuelas y se aplicó a aquellos alumnos que aceptaron participar voluntariamente. Se le comunicó al grupo de alumnos que se intentaba exclusivamente de un diagnóstico de su salud reproductiva, que el cuestionario era de autollenado, anónimo y estrictamente confidencial, que no se daría ninguna orientación personalizada.

El cuestionario constó de nueve secciones; las más importantes se refieren a temas de educación sexual, sexualidad, embarazo y comportamientos de riesgo de los adolescentes y violencia familiar. Las principales preguntas fueron las siguientes: ¿Cuál es la opinión de los estudiantes respecto a la violencia contra las mujeres? ¿Existen diferencias entre las percepciones de los estudiantes hombres y mujeres de la violencia en sus hogares? ¿Quiénes son los mayores protagonistas de esta violencia? ¿Existen diferencias en el tipo de agresor, según el sexo del adolescente agredido?

Únicamente el $0.7 \%$ se negó a contestar el cuestionario y el $6.9 \%$ no lo contestó completo, por lo que la muestra final de alumnos fue de 3,439 en Chiapas y 2,543 en SLP. El trabajo de campo se realizó en Chiapas de marzo a junio del 2003 y en SLP de octubre a noviembre del mismo año.

Para realizar el análisis de la información, se seleccionaron aquellas variables que podían caracterizar demográfica, económica y socialmente a la población.

Con base a la información disponible, se efectuó un análisis bivariado de datos utilizando la prueba de la ji-cuadrada. Para conocer algunos de los determinantes de la agresión familiar se efectuó un análisis bivariado y multivariado de datos mediante la regresión logística lineal tomando en consideración el efecto del diseño muestral. Para tal fin se utilizó el paquete informático Stata v.9. La variable dependiente consideró si hubo o no agresión por parte de algún familiar hacia el estudiante. Las variables independientes fueron el sexo del alumno, la edad, si hablaban alguna lengua indígena, si el alumno había tomado alcohol durante los tres meses anteriores a la encuesta o si había alguna vez consumido una droga, el tamaño de localidad donde se ubica la escuela y finalmente si la madre participaba en las decisiones importantes en el hogar. 
Tabla 1 - Características generales de los alumnos entrevistados. M éxico, 2003.

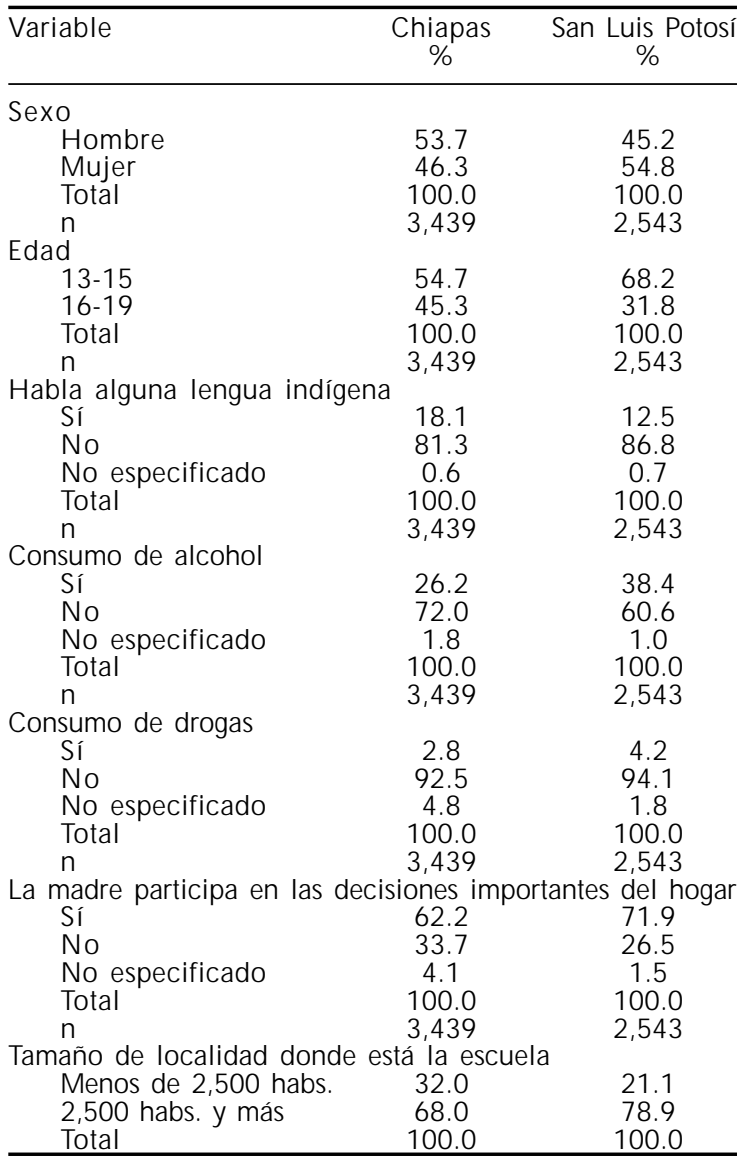

El protocolo de investigación cumplió con todos los aspectos éticos requeridos y fue aprobado por El Programa de Estudios en Población y Procesos Urbanos del Centro Regional de Investigaciones Multidisciplinarias de la Universidad Nacional Autónoma de México.

\section{RESU LTADOS}

Se obtuvo una proporción ligeramente mayor de varones entrevistados en Chiapas (53.7\%) que de mujeres $(46.3 \%)$ y en SLP por el contrario se entrevistaron más mujeres (54.8\%). El $18.1 \%$ de los estudiantes chapanecos declaró hablar alguna lengua indígena, mientras que en SLP únicamente el $12.5 \%$. Se encontró un mayor consumo de alcohol en SLP que en Chiapas (38.4\% vs $26.2 \%$ ) y este mismo comportamiento también se obtuvo en el consumo de drogas (4.2\% vs 2.8\%). En Chiapas, un $32.0 \%$ de las escuelas estaban situadas en áreas menores de 2,500 habitantes y $21.1 \%$ en SLP (Tabla 1). En los siguientes apartados se procederá a responder las preguntas anteriormente planteadas.

El estudio buscó conocer la opinión que los estudiantes tienen sobre la violencia ejercida hacia las mujeres. La mayoría de los hombres expresaron que no estaban de acuerdo con pegarle a una mujer en caso de tener un conflicto con ella. Sin embargo, un $11 \%$ respondió lo contrario en ambos estados. Situación contrastante con lo que respondieron las mujeres, ya que sólo un $2.7 \%$ en Chiapas y un $1.6 \%$ en SLP aceptaría, en teoría, ser agredida.

Con el propósito de ver si la violencia es diferencial por sexo, se analizó la información sobre las agresiones que se cometieron hacia los estudiantes por parte de algún familiar.

La primera pregunta efectuada a este respecto fue averiguar cuántos estudiantes habían sido agredidos en algún pleito o pelea por alguno(s) miembro(s) de su familia. El 14\% de los estudiantes de Chiapas y el $19 \%$ de los estudiantes de SLP reportó haber sufrido una agresión por parte de un familiar. Si se analiza la información por sexo, el haber sido víctima de una agresión resultó significativamente más frecuente en las alumnas que en los alumnos (en Chiapas, 17.6\% de las mujeres y $11.1 \%$ de los hombres; en SLP $20.9 \%$ de las mujeres y $16.8 \%$ de los hombres). Además, en las dos entidades analizadas se encontró una mayor proporción de estudiantes de 16 a 19 años, de los dos sexos, que reportaron haber sido agredidos por algún

Tabla 2 - Alumnos que han sido agredidos en algún pleito o pelea por algún miembro de la familia, según sexo y grupos de edad. México, 2003

\begin{tabular}{|c|c|c|c|c|c|c|}
\hline Respuestas & 13 a $\underset{\%}{15}$ años & $\begin{array}{c}\text { Chiapas } \\
16 \text { a } 19 \text { años } \\
\%\end{array}$ & $\begin{array}{c}\text { Total } \\
\%\end{array}$ & 13 a $\underset{\%}{15}$ años & $\begin{array}{c}\text { San Luis Potosí } \\
16 \text { a } 19 \text { años } \\
\%\end{array}$ & $\begin{array}{c}\text { Total } \\
\%\end{array}$ \\
\hline $\begin{array}{c}\text { Hombres } \\
\text { Sí } \\
\text { No } \\
\text { Total } \\
n\end{array}$ & $\begin{array}{c}9.1 \\
90.9 \\
100.0 \\
906\end{array}$ & $\begin{array}{c}13.4 \\
86.6 \\
100.0 \\
869 \\
\mathrm{X}^{2}=5,493 \quad \mathrm{p}<0.05\end{array}$ & $\begin{array}{c}11.1 \\
88.9 \\
100.0 \\
1,775\end{array}$ & $\begin{array}{c}13.3 \\
86.7 \\
100.0 \\
725\end{array}$ & $\begin{array}{c}24.3 \\
75.7 \\
100.0 \\
468 \\
\mathrm{X}^{2}=9,759 \mathrm{p}<0.005\end{array}$ & $\begin{array}{l}16.8 \\
83.2 \\
100.0 \\
1,193\end{array}$ \\
\hline $\begin{array}{l}\text { Mujeres } \\
\text { Sí } \\
\text { No } \\
\text { Total } \\
n\end{array}$ & $\begin{array}{c}16.3 \\
83.7 \\
100.0 \\
910\end{array}$ & $\begin{array}{c}19.3 \\
80.7 \\
100.0 \\
754 \\
\mathrm{X}^{2}=3,440 \mathrm{p}>0.05\end{array}$ & $\begin{array}{c}17.6 \\
82.4 \\
100.0 \\
1,664\end{array}$ & $\begin{array}{c}15.3 \\
84.7 \\
100.0 \\
869\end{array}$ & $\begin{array}{c}32.9 \\
67.1 \\
100.0 \\
481 \\
X^{2}=25,773 p=0.000\end{array}$ & $\begin{array}{r}20.9 \\
79.1 \\
100.0 \\
1,350\end{array}$ \\
\hline
\end{tabular}


familiar, siendo estadísticamente significativo para los dos sexos en SLP y en los hombres chapanecos. En el caso de las mujeres chapanecas fue marginalmente significativo $(\mathrm{p}=0.064)($ Tabla 2$)$.

Con respecto a la fecha de la última agresión cometida contra los estudiantes, el $56 \%$ de los de Chiapas y $63 \%$ de los de SLP fueron agredidos por algún(os) familiar(es) el mismo año de la entrevista.

En el último pleito, la principal persona que agredió a los estudiantes fue el padre en Chiapas y en SLP fueron los hermanos (el padre participó en el $38.0 \%$ de los casos en Chiapas y en el $31.9 \%$ de los pleitos en SLP, y los hermanos $24.5 \%$ en Chiapas y $36.9 \%$ en SLP). La madre tuvo una participación en la violencia familiar mucho menor en ambos estados. El resto de los agresores se repartió entre otros familiares y no familiares de los estudiantes que viven en el hogar (Figura 1).

Si bien en SLP los estudiantes declararon mayor violencia que en Chiapas, la naturaleza del agresor fue diferente en ambos estados ya que los estudiantes reportaron una participación de la madre en la violencia familiar mucho menor que la del padre o la de los hermanos; la participación materna fue todavía menos frecuente en Chiapas que en SLP.

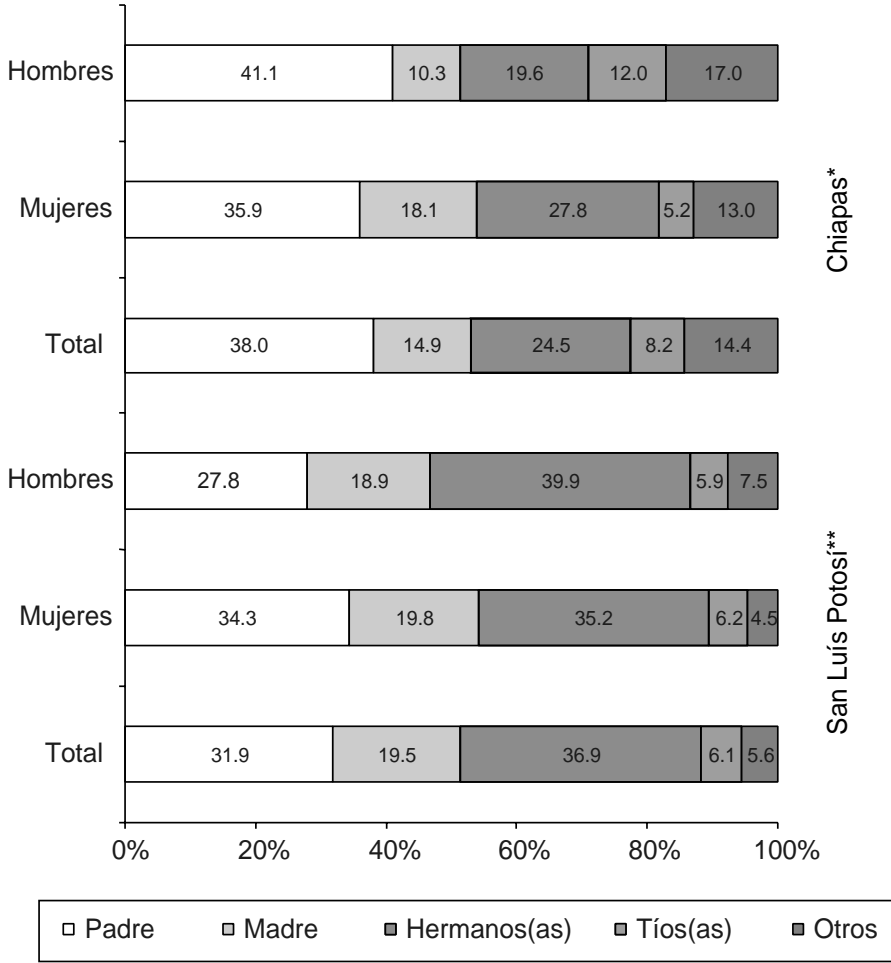

${ }^{*} X^{2}=17.09 ; p<0.05 ; * * X^{2}=4.33 ; p>0.05$

Figura 1 - Porcentaje de estudiantes que fueron agredidos en el hogar, según personassoa que los agredió enm la última pelea. México, 2003.

Centrándonos en las diferencias del tipo de agresor según el sexo del estudiante, la participación de la madre en las agresiones a los jóvenes fue mayor hacía las mujeres adolescentes que hacia los varones en el estado de Chiapas. Así, un $18.1 \%$ de las alumnas y un

Tabla 3 - Agresión hacia los estudiantes adolescentes por parte de un familiar: razones de momios crudas y ajustadas* según características seleccionadas. M éxico, 2003.

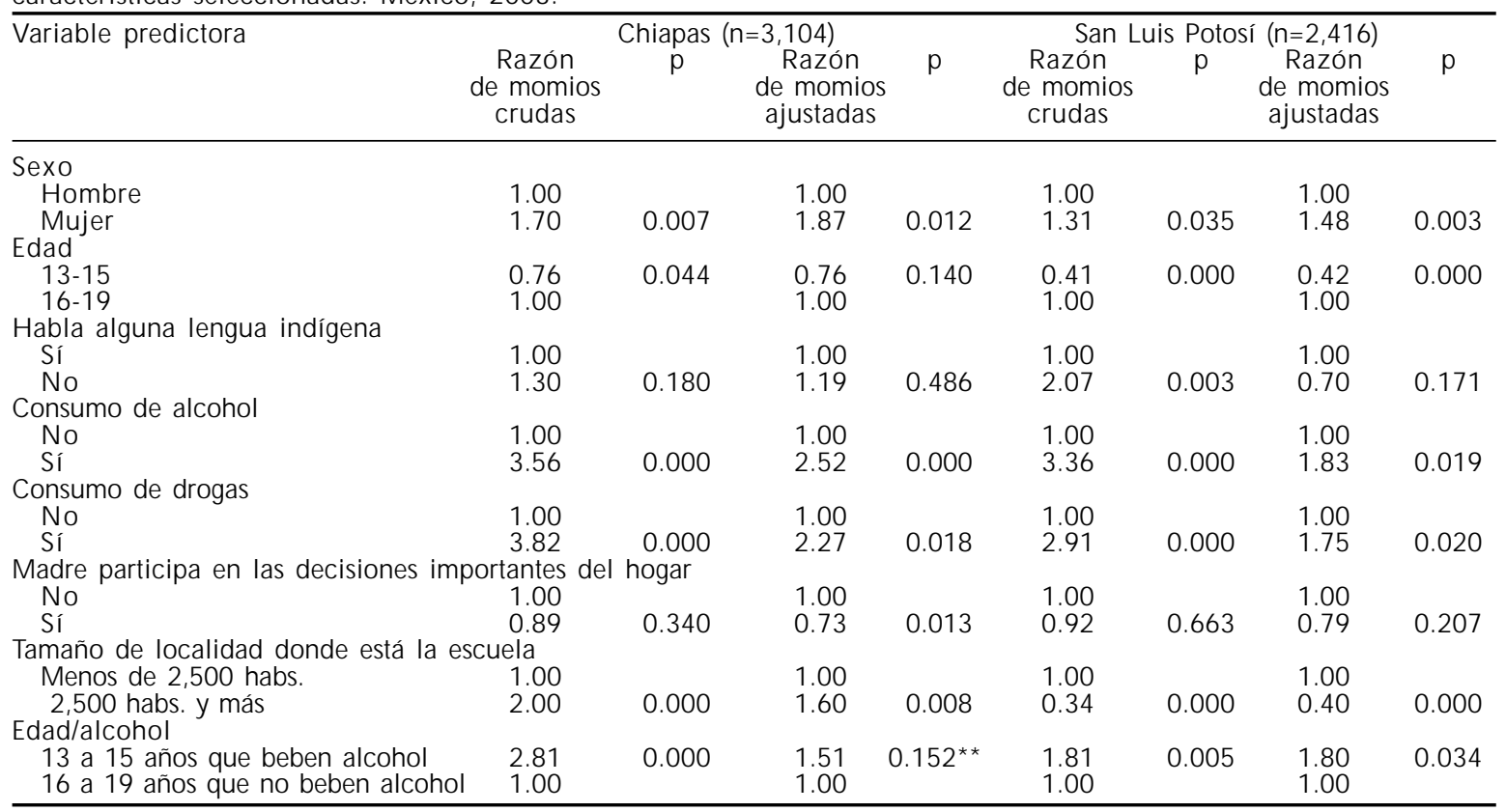

*Ajustadas por todas las variables

**Valor estadísticamente significativo cuando se trata de una interacción 


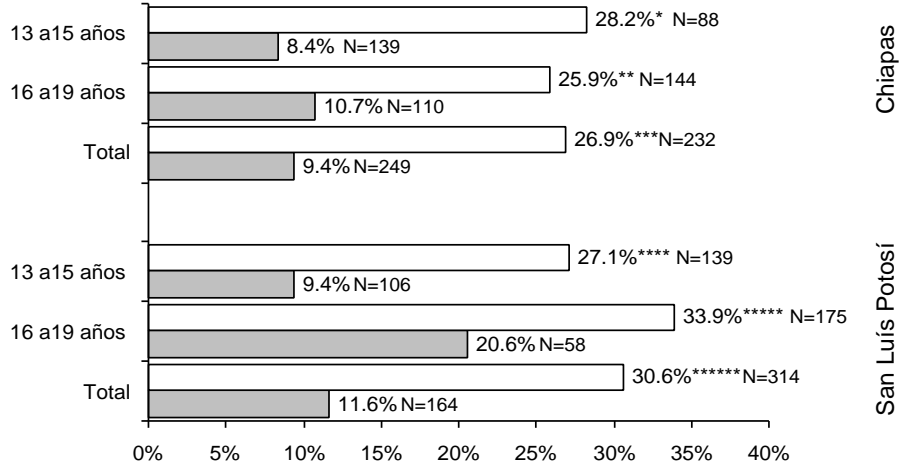

$\square$ No bebe alcohol $\square$ Sí bebe alcohol

$\begin{array}{ll}* X^{2}=77.13 ; p<0.01 & * * * * X^{2}=92.59 ; p<0.01 \\ * * X^{2}=74.32 ; p<0.01 & * * * * X^{2}=25.48 ; p<0.01 \\ * * * X^{2}=158.46 ; p<0.01 & * * * * * X^{2}=136.24 ; p<0.01\end{array}$

Figura 2 - Porcentaje de estudiantes de 13 a 19 años de edad agredidos por un familiar según si beben alcohol. México, 2003.

$10.3 \%$ de los alumnos reportaron haber sido agredidos por la madre.También las alumnas reportaron una mayor violencia hacia ellas por parte de los hermanos(as) y menor de los tíos(as). En el caso de SLP, las diferencias entre sexos y el tipo de agresor no resultaron significativas (Figura 1).

Del análisis bivariado se obtuvo que en las dos entidades federativas, las variables sexo del entrevistado, la edad, el consumo del estudiante de alcohol o de drogas, el lugar donde se ubica la escuela, y la interacción de la edad con el alcohol son las que más exponen a los estudiantes al riesgo de violencia (Tabla 3 ).

De esta forma, el ser mujer expone a las estudiantes a mayor violencia en el hogar que a los hombres (Chiapas: $\mathrm{RM}=1.70, \mathrm{p}=0.007$; SLP: $\mathrm{RM}=1.31$, $\mathrm{p}=0.035)$. El haber consumido alcohol en los tres meses anteriores a la encuesta (Chiapas: $\mathrm{RM}=3.56$, $\mathrm{p}=0.000$; SLP: $\mathrm{RM}=3.36, \mathrm{p}=0.000$ ) es una de las variables que más expone a los estudiantes a la violencia familiar. Al parecer, la presencia del alcohol en el hogar se relaciona de manera importante con la agresión familiar; de hecho, es la variable de mayor riesgo en ambos estados. Desafortunadamente en la encuesta no se contó con el consumo de alcohol de los demás miembros del hogar, pero queda claro que este elemento debe ser considerado en los análisis de violencia El haber consumido alguna vez drogas (Chiapas: $\mathrm{RM}=3.82$, $\mathrm{p}=0.000$; SLP: $\mathrm{RM}=2.91, \mathrm{p}=0.000$ ) también expone fuertemente a los alumnos a sufrir violencia por parte de un familiar. El vivir en las áreas urbanas en Chiapas los expone al doble de violencia que los que viven en áreas rurales $(\mathrm{p}=0.000)$. En SLP el no hablar una lengua indígena los expone al doble de violencia que los que son bilingües ( $\mathrm{p}=0.003$ ), y muy al contrario de lo que sucede en Chiapas, el vivir en un área

urbana los protege de la violencia en el hogar $(\mathrm{RM}=0.34, \mathrm{p}=0.000)$.

En vista de que el uso del alcohol obtuvo la razón de momios más alta, y considerando la importancia de la edad en esta problemática, se revisó la relación entre la agresión intrafamiliar la edad y el consumo de alcohol (Figura 2). La violencia fue claramente mayor en aquellos estudiantes que beben alcohol en comparación con los que no lo hacen. Las diferencias se amplían en los grupos de edad más jóvenes y son estadísticamente significativas. En el análisis bivariado se obtuvo que el tener de 13 a 15 años y haber bebido alcohol los tres meses anteriores a la encuesta los expone a sufrir tres veces más violencia en Chiapas $(\mathrm{p}=0.000)$ y dos veces más violencia en SLP $(p=0.005)$ en comparación con los que tienen de 16 a 19 años y no bebieron alcohol en este tiempo (Tabla 3).

Al efectuar el análisis multivariado de datos, ajustado por todas las variables consideradas en el análisis bivariado, se obtuvo que en Chiapas las variables que arriesgan al estudiante a la violencia familiar son el sexo, el alcohol, la droga, y el que la escuela este ubicada en un entorno urbano (Tabla 3). De esta manera el ser estudiante mujer la arriesga 1.87 veces frente al sexo masculino a recibir alguna agresión por parte de un familiar ( $\mathrm{p}=0.012$ ), esto confirma la mayor vulnerabilidad femenina frente a la violencia familiar. El haber bebido alcohol tres meses antes de la encuesta arriesga a los alumnos en 2.52 veces más $(p=0.000)$. Y el consumo de droga también resultó muy importante ya que arriesga a los alumnos en 2.27 veces $(\mathrm{p}=0.018)$ a sufrir violencia por parte de un familiar. Finalmente, el que la escuela se encuentre en un contexto urbano arriesga en 1.60 veces a los alumnos $(p=0.008)$. La interacción de la edad con el alcohol resultó ser significativa, esto es, encontramos que cuando los alumnos tienen de 13 a 15 años y beben tienen una probabilidad de sufrir una agresión por parte de algún familiar de 1.51 veces respecto a los que no beben y tienen de 16 a 19 años de edad. Por otra parte, la variable protectora en Chiapas fue el que la madre participe en las decisiones del hogar $(\mathrm{RM}=0.73, \mathrm{p}=0.013)$. Esto puede deberse a que cuando se incrementa la equidad entre géneros, existe una mayor democratización de la familia y por ende, una menor violencia familiar.

El modelo ajustado de regresión logística aplicado a SLP tuvo básicamente resultados similares que los de Chiapas (Tabla 3), excepto en el caso del lugar donde se encontraba ubicada la escuela y la edad del estudiante. Así se encontró que en SLP también el ser mujer 
( $\mathrm{RM}=1.48, \mathrm{p}=0.003)$, el haber bebido alcohol $(\mathrm{RM}=1.83, \mathrm{p}=0.019)$ o consumir droga alguna vez $(\mathrm{RM}=1.75, \mathrm{p}=0.020)$ los arriesga a mayor violencia por parte de algún familiar. Asimismo, el ser joven $(\mathrm{RM}=0.42$, $\mathrm{p}=0.000)$ o que la escuela se encuentre en un contexto urbano $(\mathrm{RM}=0.40, \mathrm{p}=0.000)$ los protege contra la violencia. La asociación de la edad con el alcohol resultó ser también significativa en SLP; de esta forma los que sufren mayor riesgo son los alumnos más jóvenes que han tomado alcohol frente a los de mayor edad que nunca han tomado $(\mathrm{RM}=1.80, \mathrm{p}=0.034)$.

\section{DISCUSIÓN}

Esta investigación tiene algunas limitaciones inherentes al diseño del estudio en el cual se obtuvo la información, y que es necesario tomar en consideración. Se trata de un estudio transversal, por lo cual no se puede evaluar la asociación entre la causa y el efecto, ya que la exposición y el evento fueron medidos al mismo tiempo. Además, la población de estudio se restringió a estudiantes, por tanto, las conclusiones del mismo deben limitarse a esta población. Por otro lado, al ser un cuestionario de autollenado, los resultados sobre un tema tabú como lo es la violencia intrafamiliar y otras características asociadas a ella pueden estar subestimados. Cabe aclarar que esta encuesta estudia la violencia familiar en un contexto de la salud reproductiva de los adolescentes en México, por lo que los resultados expresan una de las primeras aproximaciones al fenómeno.

A pesar de que la violencia contra las mujeres no parece tener aceptación entre la gran mayoría de los estudiantes, los adolescentes, mujeres y hombres, continúan siendo hoy en día una de las poblaciones más vulnerables debido a su dependencia física, afectiva, económica y social hacia los adultos a su cargo. Esta circunstancia los convierte en blanco fácil de una gran gama de abusos perpetrados en contra de su integridad como seres humanos. ${ }^{22}$

A lo largo de todo el trabajo se encontró que sistemáticamente las mujeres adolescentes reportaron mayor violencia familiar que los varones, también fue encontrado en otras investigaciones. ${ }^{2,8}$ En el caso de Chiapas, tanto el padre como la madre ejercieron una violencia notablemente superior hacía las hijas que hacia los hijos. Esto refleja que no sólo los varones, sino también las mujeres reproducen la desigualdad entre los sexos, ya que las madres chapanecas ejercen mayor violencia hacia el sexo femenino. En estudios recientes, se ha encontrado un incremento de la exposición de los hijos a agresiones maternas. ${ }^{13,19}$

En ambos estados la violencia ejercida por el padre fue mucho mayor que la ejercida por la madre. El hecho de que en Chiapas se encontró mayor participación del padre en la agresión familiar que en SLP parece reflejar una sociedad chapaneca jerárquica, y un ambiente dónde muchos de los jóvenes sufren la violencia. Así, la violencia ejercida por el padre es una estrategia de mayor empoderamiento, es el resultado de un desequilibrio de poder en las relaciones hombre-mujer. Esta desigualdad se da a partir de la identidad genérica en un entorno patriarcal asimétrico. ${ }^{25}$

Por otro lado, al parecer, un gran número de jóvenes está en contra de la violencia hacia las mujeres; sin embargo, al vivirla desde muy pequeños en sus hogares, es muy probable que la reproduzcan, como un patrón de conducta aprendido. Esta transmisión intergeneracional de la violencia se explica como resultado de este aprendizaje. ${ }^{1,20}$ A veces los menores de edad no son víctimas directas de acciones violentas, sino observadores dentro del hogar, sobre todo entre sus padres. Así aprenden que las faltas de respeto y los golpes son medios válidos para dirimir diferencias a costa de la integridad física y emocional. ${ }^{6,15}$ Esta situación se da de manera más evidente en Chiapas que en SLP, aunque en SLP un mayor número de estudiantes declaró haber recibido alguna agresión familiar. Es posible que en este estado exista una mejor declaración de la violencia ya que se trata de una entidad menos marginada que Chiapas.

Los resultados del presente estudio refuerzan la urgente necesidad de seguir profundizando en los análisis de violencia familiar, cometida contra la población más vulnerable, es decir, la violencia que se ejerce contra mujeres, niños y adolescentes. Es necesario romper el círculo vicioso sobre la violencia vivida por los adolescentes desde muy jóvenes que tiene como consecuencia que cuando estos sean adultos maltraten a su vez a sus hijos. ${ }^{17}$

Se puede decir que los modelos de regresión logística confirman la mayor vulnerabilidad femenina frente a la violencia familiar en ambas entidades federativas.

También se encontró mayor violencia en las áreas urbanas en Chiapas y en las rurales en SLP; esto puede deberse a que los alumnos del área rural que asisten a secundaria y preparatoria en Chiapas son una población selecta, ya que un porcentaje elevado de personas en el campo no llega hasta esos niveles de escolaridad.

Asimismo, el que los estudiantes beban alcohol o consuman drogas resultaron ser las dos variables más importantes que exponen a los estudiantes a la violencia intrafamiliar en ambos estados confirmado en otras investigaciones, ${ }^{10}$ aunque sigue siendo mayor 
el riesgo en Chiapas. También es relevante el papel de la madre en las decisiones importantes en el hogar para que exista menor violencia en las familias chapanecas.

El consumo de alcohol de los más jóvenes aumenta el riesgo de mayor violencia en el hogar. Esto puede deberse a que el consumo de alcohol a edades muy tempranas refleja hogares con hábitos menos favorables a la armonía familiar. ${ }^{16}$ Esto lleva a reflexionar si es el haber vivido la violencia desde pequeños la que origina que algunos alumnos tengan problemas de alcoholismo o drogadicción, o si son estos problemas los que originan (o agudizan) la violencia intrafamiliar. Lo que no hay duda es que la presencia de alcohol y de drogas en el hogar se relaciona de manera importante con la violencia familiar. Algunos estudios han dado cuenta de que los padres que abusan del alcohol o drogas son violentos con sus hijos. ${ }^{17}$ Quizás, la conducta aprendida en

\section{REFERENCIAS}

1. Anda RF, Felitti VJ, Chapman DP, Croft JB, Williamson DF, Santelli J, et al. Abused boys, battered mothers, and male involvement in teen pregnancy. Pediatrics. 2001;107:19-25.

2. American Medical Association. Adolescents as victims of family violence. JAMA. 1993;270:1850-6.

3. Brook DW, Brook JS, Rosen Z, De la Rosa M, Montoya ID, Whiteman M. Early risk factors for violence in Colombia adolescents. Am J Psychiatry. 2003;160:1470-8.

4. Cohen DA, Richardson J, LaBree L. Parenting behaviors and the onset of smoking and alcohol use: a longitudinal study. Pediatrics. 1999;103:368-75.

5. Dembo R, Williams L, Wothke W, Schmeidler J, Brown $\mathrm{CH}$. The role of family factors, physical abuse, and sexual victimization experiences in high-risk youth's alcohol and other drug use and delinquency: a longitudinal model. Violence Vict. 1992;7:245-66.

6. DuRant RH, Treiber F, Goodman E, Woods ER. Intentions to use violence among young adolescents. Pediatrics. 1996;(6 Pt 1):1104-8.

7. DuRant RH, Altman D, Wolfson M, Barkin S, Kreiter $S$, Krowchuk D. Exposure to violence and victimization, depression, substance use, and the use of violence by young adolescents. J Pediatr. 2000;137:707-13.

8. Eamon MK. Antecedents and socioemotional consequences of physical punishment on children in two-parent families. Child Abuse $\mathrm{Negl}$. 2001;25:787-802. casa, hace más propensos a los adolescentes a repetir el comportamiento de beber alcohol o drogarse. Por contraparte, otras investigaciones dan cuenta de que aquellos adolescentes que dicen interesarles a sus padres, tienen cercanía y comunicación con ellos, además de sentir el respeto y amor familiar están menos propensos a alcohol y drogas., ${ }^{418} \mathrm{De}$ hecho, en un estudio realizado en Brasil, se encontró que los hijos proponen evitar el uso de alcohol y de drogas en casa, como una medida importante para disminuir o acabar con la violencia familiar. ${ }^{12}$ Otros factores de riesgo son el acceso fácil al alcohol, tabaco y drogas en casa, ya que los adolescentes tendrán una mayor propensión a utilizarlos. ${ }^{17}$

Finalmente, hacen falta en México investigaciones que analicen la violencia contra los adolescentes. Se cree que los hallazgos del presente estudio pueden sugerir algunos caminos para el diseño de una encuesta nacional.
9. Ellickson P, Saner H, McGuigan KA. Profiles of violent youth: substance use and other concurrent problems. Am J Public Health. 1997:87:985-91.

10. Freudenberg $N$, Roberts L, Richie BE, Taylor RT, McGillicuddy K, Greene MB. Coming up in the boogie down: the role of violence in the lives of adolescents in the South Bronx. Health Educ Behav. 1999;26:788-805.

11. Garza-Aguiar J, Díaz-Maciel E. Elementos para el estudio de la violación sexual. Salud Pública Méx. 1997;39:539-45.

12. Moreira EC, Centa ML. 0 retrato da violência domiciliar para crianças de 8 a 11 anos. Fam Saúde Desenvolv. 2003;5:35-42.

13. Mohr WK, Noone-Lutz MJ, Fantuzzo JW, Perry MA. Children exposed to family violence: a review of empirical research from a developmental-ecological perspective. Trauma Violence Abuse. 2000;1:264-83.

14. Organización Panamericana de la Salud. Informe mundial sobre la violencia y la salud. Washington (DC); 2003. [Publicación Científica y Técnica, 588]

15. Orpinas $P$, Murray $N$, Kelder $S$. Parental influences on students' aggressive behaviors and weapon carrying. Health Educ Behav. 1999;26:774-87.

16. Ramos-Lira L, Saldívar-Hernández G, Medina-Mora $M E$, Rojas-Guiot E, Villatoro-Velázquez J. Prevalencia de abuso sexual en estudiantes y su relación con el consumo de drogas. Salud Pública Méx. 1998:40:221-33. 
17. Resnick MD, Bearman PS, Blum RW, Bauman KE $\mathrm{H}$ arris KM, Jones J, et al. Protecting adolescents from harm: findings from the national longitudinal study on adolescent health. JAMA. 1997;278:823-32.

18. Simons-Morton B, Haynie DL, Crump AD, Eitel $P$, Saylor KE. Peer and parent influences on smoking and drinking among early adolescents. Health Educ Behav. 2001;28:95-107.

19. Straus MA. Spanking and the making of violent societies. Pediatrics. 1996:98(4 Pt 2):837-42.

20. Thomas SB, Leite B, Duncan T. Breaking the cycle of violence among youth living in metropolitan Atlanta: a case history of kids alive and loved. Health Educ Behav. 1998;25:160-74.

21. Thompson RA, Christiansen EH, Jackson S, Wyatt JM, Colman RA, Peterson RL, et al. Parent attitudes and discipline practices: profiles and correlates in a nationally representative sample. Child Maltreat. 1999:4:316-30.
22. Valdez R, Hijar M. Del silencio privado a las salas de urgencias: la violencia familiar como un problema de salud pública. Gac Méd Méx. 2002;138(2):159-63.

Disponible en http://www.medigraphic.com/espanol/ e-htms/e-gaceta/e-gm2002/e-gm02-2/emgm022e.htm [acceso en 5 ago 2006]

23. Van der Wal MF, Wit CA, Hirasing RA. Psychosocial health among young victims and offender of direct and indirect bullying. Pediatrics. 2003;111(6 Pt 1):1312-7.

24. Vicary JR, Lerner JV. Parental attributes and adolescent drug use. J Adolesc. 1986;9:115-22.

25. Villaseñor-Farías M, Castañeda-Torres JD. Masculinidad, sexualidad, poder y violencia: análisis de significados en adolescentes. Salud Pública Méx. 2003;45(Supl 1):S44-57.

Financiada por la Fundación David and Lucile Packard y llevada a cabo en el Centro Regional de Investigaciones Multidisciplinarias, Universidad Nacional Autónoma de México (CRIM/U NAM).

Documento presentado en la Sesión: Género, Violencia y Salud Reproductiva (GEN 004) en la Reunión de la Asociación de Estudios Latinoamericanos, Las Vegas, Nevada, 7 al 9 de 0 ctubre del 2004. 